\title{
Effect of ventilator-induced lung injury on the development of reperfusion injury in a rat lung transplant model
}

\author{
Marc de Perrot, MD \\ Yumiko Imai, $\mathrm{MD}^{\mathrm{b}}$ \\ George A. Volgyesi, MD ${ }^{b}$ \\ Thomas K. Waddell, MD \\ Mingyao Liu, MD \\ J. Brendan Mullen, $\mathrm{MD}^{\mathrm{C}}$ \\ Karen McRae, $\mathrm{MD}^{\mathrm{d}}$ \\ Haibo Zhang, $\mathrm{MD}^{\mathrm{b}}$ \\ Arthur S. Slutsky, MD \\ V. Marco Ranieri, MD \\ Shaf Keshavjee, MD
}

From the Thoracic Surgery Research Laboratory, ${ }^{a}$ Toronto General Hospital, Respiratory Medicine and Critical Care, ${ }^{\mathrm{b}} \mathrm{St}$ Michael Hospital, Department of Pathology and Laboratory Medicine, ${ }^{\mathrm{c}}$ Mount Sinai Hospital, Department of Anesthesia, ${ }^{\mathrm{d}}$ Toronto General Hospital, University of Toronto, Toronto, Ontario, Canada, and the Dipartimento di Discipline Medico-Chirurgiche, ${ }^{\mathrm{e}}$ Sezione di Anestesiologia e Rianimazione, Università di Torino, Ospedale S. Giovanni Battista, Torino, Italy.

Received for publication Nov 8, 2001; revisions requested Feb 5, 2002; revisions received March 11, 2002; accepted for publication March 24, 2002.

Address for reprints: S. Keshavjee, MD, Director, Thoracic Surgery Research Laboratory, Toronto General Hospital, 200 Elizabeth St, EN 10-224, Toronto, Ontario, Canada M5G 2C4 (E-mail: shaf.keshavjee @uhn.on.ca).

J Thorac Cardiovasc Surg 2002;124: 1137-44

Copyright () 2002 by The American Association for Thoracic Surgery

0022-5223/2002 $\$ 35.00+0 \quad \mathbf{1 2 / 1 / 1 2 5 0 5 6}$

doi: $10.1067 / \mathrm{mtc} .2002 .125056$
Objective: Although mechanical ventilation can potentially worsen preexisting lung injury, its importance in the setting of lung transplantation has not been explored. This study was undertaken to examine the effect of 2 ventilatory strategies on the development of ischemia-reperfusion injury after lung transplantation.

Methods: In a rat lung transplant model animals were randomized into 2 groups defined by the ventilatory strategy during the early reperfusion period. In conventional mechanical ventilation the transplanted lung was ventilated with a tidal volume equal to $50 \%$ of the inspiratory capacity of the left lung and a low positive end-expiratory pressure. In minimal mechanical stress ventilation the transplanted lung was ventilated with a tidal volume equal to $20 \%$ of the inspiratory capacity of the left lung, and positive end-expiratory pressure was adjusted according to the shape of the pressure-time curve to minimize pulmonary stress.

Results: After 3 hours of reperfusion, oxygenation from the transplanted lung was significantly higher with minimal mechanical stress ventilation than with conventional ventilation. In addition, elastance, cytokine levels, and morphologic signs of injury were significantly lower in the group with minimal mechanical stress ventilation.

Conclusions: This study demonstrates that the mode of mechanical ventilation used in the early phase of reperfusion of the transplanted lung can influence ischemiareperfusion injury, and a protective ventilatory strategy on the basis of minimizing pulmonary mechanical stress can lead to improved lung function after lung transplantation.

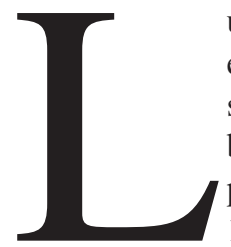

ung transplantation has evolved over the last 2 decades from an experimental treatment to become the mainstay of therapy for endstage lung diseases. Approximately 10,000 lung transplants have been performed worldwide, and more than 1000 transplants are performed annually. ${ }^{1}$ Despite the success of lung transplantation, $10 \%$ to $20 \%$ of the patients have poor immediate graft function. These patients require prolonged ventilatory support and have a postoperative mortality rate of $40 \%$ to $60 \%$, which is dramatically higher than in the uncomplicated lung transplant group. ${ }^{2,3}$ In addition, there is evidence suggesting that severe 
reperfusion injury is also associated with an increased risk of acute rejection and graft dysfunction in the long term. ${ }^{4,5}$

Mechanical ventilation maintains adequate systemic oxygenation, allows the respiratory muscles to rest, and delays mortality in many patients with acute lung injury (ALI). However, a number of animal and clinical studies have shown that mechanical ventilation can worsen preexisting lung injury or produce ALI de novo in previously normal lungs. ${ }^{6}$ The postulated mechanism responsible for ventilator-induced lung injury (VILI) is the mechanical stress induced by shear forces that might initiate or worsen pulmonary inflammatory processes and alter the alveolar-endothelial barrier, as well as the surfactant function. ${ }^{6-8}$ In a nonuniformly expanded lung, tidal recruitment of collapsed regions surrounded by open alveoli or overdistention of normal alveoli close to collapsed areas, or both might generate forces dramatically higher than the measured transpulmonary pressure $\left(\mathrm{P}_{\mathrm{L}}\right){ }^{9}$

The pressure-volume $(\mathrm{P}-\mathrm{V})$ curve of the respiratory system is characterized by a sigmoid shape, with a lower inflection point (LIP) corresponding to the pressure at which collapsed alveoli begin to be recruited and an upper inflection point (UIP) corresponding to the volume at which alveolar overdistention might begin to occur. Protective ventilatory approaches on the basis of the P-V curve have been designed to minimize the effects of mechanical stress on pulmonary and systemic inflammatory responses and to improve outcomes. By using this curve, the level of positive end-expiratory pressure (PEEP) is set above the LIP to avoid cycling end-expiratory alveolar collapse, and the tidal volume $\left(\mathrm{V}_{\mathrm{T}}\right)$ is set to maintain the end-inspiratory pressure and volume at less than the UIP to avoid alveolar overdistention. ${ }^{10-12}$ However, the clinical use of the static P-V curve has been limited by the complexity of its measurement and interpretation. ${ }^{13,14}$

The dynamic airway opening pressure-time (P-t) profile during constant-flow inflation might be used to set a protective ventilatory strategy allowing breath-by-breath assessment to minimize mechanical stress, as recently shown in an animal model of ALI. A computerized mathematic equation permits quantification of the shape of the P-t curve by a coefficient termed the stress index. ${ }^{15,16}$ Stress index values of less than 1 indicate a P-t curve characterized by a downward concavity corresponding to a static P-V curve with a distinct LIP; this ventilatory pattern was associated with VILI because of mechanical stress caused by continuous opening and closing of the collapsed alveolar units. Stress index values of greater than 1 indicate a P-t curve characterized by an upward concavity corresponding to a static P-V curve with a distinct UIP; animals ventilated with this ventilator pattern showed evidence of VILI as a result of mechanical stress caused by overdistention of the alveolar units. Stress index values of 1 describe a straight P-t curve corresponding to a linear static P-V curve; this ventilatory pattern produces minimal mechanical stress and no VILI because neither overdistention nor alveolar collapse occurs with mechanical ventilation.

In human lung transplantation the majority of patients require mechanical ventilatory support for at least several hours in the period immediately after lung transplantation. Although necessary, this period of positive-pressure ventilation could potentially increase the injury to the transplanted lung if the ventilatory strategy used increases pulmonary stresses, particularly in patients with the most severe form of ischemia-reperfusion (I-R) injury. Although the protective ventilatory strategies have been shown to improve the outcome of patients with ALI, ${ }^{10-12}$ the effect of ventilatory strategies on the development of pulmonary injury in lung transplantation has not been explored. This study tested the hypothesis that mechanical ventilation might worsen reperfusion injury after lung transplantation. We evaluated whether a ventilatory strategy aimed at minimizing mechanical stress would minimize pathophysiologic indices of ALI in the setting of lung transplantation.

\section{Material and Methods}

\section{Animals}

Experiments were performed with male Sprague-Dawley rats weighing 400 to $450 \mathrm{~g}$ (Charles River Inc, Montreal, Quebec, Canada). All animals received care in compliance with the Principles of Laboratory Animal Care formulated by the National Society for Medical Research, the Guide for the Care and Use of Laboratory Animals (National Institutes of Health publication no. 85-23, Revised 1985), and the Guide to the Care and Use of Experimental Animals formulated by the Canadian Council on Animal Care. The experimental protocol was approved by the Animal Care Committee of the Toronto General Hospital Research Institute.

\section{Lung Transplantation Procedure}

Harvest and storage. Donor rats were anesthetized by means of an intraperitoneal injection of $1 \mathrm{~mL}$ of sodium pentobarbital (Somnotol; MTC Pharmaceuticals, Cambridge, Ontario, Canada) and intubated through a tracheostomy with a 14-gauge intravenous catheter. The tracheostomy tube was then connected to a volumecycled ventilator (Harvard Rodent Ventilator, Model 683; Harvard, South Natick, Mass) at the following ventilator settings: $\mathrm{V}_{\mathrm{T}}$ of $10 \mathrm{~mL} / \mathrm{kg}$, respiratory rate of $80 \mathrm{breaths} / \mathrm{min}$, fraction of inspiratory oxygen of 1.0, and PEEP of $2 \mathrm{~cm} \mathrm{H}_{2} \mathrm{O}$. A median laparosternotomy was then performed, and 400 USP Units of heparin (Hepalean; Organon Teknika, Toronto, Ontario, Canada) was injected into the inferior vena cava. For retrieval of the heart-lung block, the inferior vena cava was incised, the left atrial appendage was excised, and a 14-gauge intravenous catheter was placed into the main pulmonary artery (PA) through an anterior incision in the right ventricular outflow tract. The lungs were then flushed through this catheter with $20 \mathrm{~mL}$ of low-potassium dextran (LPD) preservation solution (Perfadex; Vitrolife, Uppsala, Sweden) and $0.1 \mathrm{~mL}$ of prostaglandin $\mathrm{E}_{1}(500 \mu \mathrm{g} / \mathrm{mL}$, Prostin VR; 
Upjohn, Don Mills, Ontario, Canada) from a height of $30 \mathrm{~cm}$. Immediately after flushing the lungs, the tracheostomy tube was clamped after inspiration to preserve the lungs in an inflated state. The heart-lung block was then removed and placed in LPD solution at $4{ }^{\circ} \mathrm{C}$. The left lung was prepared for transplantation with the placement of two 14-gauge cuffs into the left PA and the left pulmonary vein (PV). The right main bronchus was ligated. The left main bronchus and the trachea were kept intubated with the 14-gauge catheter for separate ventilation after the transplantation procedure. The left lung was then placed into $40 \mathrm{~mL}$ of LPD solution at $4^{\circ} \mathrm{C}$ for a total of 4 hours of cold ischemic time.

Transplantation. Recipient animals were anesthetized in a halothane chamber, and a tracheostomy was performed as described for the donor animals. The recipient animals were ventilated with a volume-cycled ventilator (Harvard Rodent Ventilator, Model 683) with a $\mathrm{V}_{\mathrm{T}}$ of $10 \mathrm{~mL} / \mathrm{kg}$, a respiratory rate 80 breaths/ min, a PEEP of $3 \mathrm{~cm} \mathrm{H}_{2} \mathrm{O}$, and a fraction of inspiratory oxygen of 1.0 , and anesthesia was maintained with $2.0 \%$ halothane. The right carotid artery was cannulated with a 20-gauge Angiocath (Deseart Medical, Inc, Becton Dickinson and Co, Sandy, Utah) to sample blood for blood gas analysis and to measure arterial blood pressure. A left thoracotomy was then performed. The left lung was mobilized by dividing the pulmonary ligament, and the hilar structures were dissected free. The left PA, PV, and main bronchus were identified and clamped with microsurgical aneurysm clamps. The left main bronchus was tied, and $\mathrm{V}_{\mathrm{T}}$, which was then limited to the right lung only, was reduced to $5 \mathrm{~mL} / \mathrm{kg}$. A ventral incision was made in the recipient PA and PV, and the cuffs of the donor lung structures were placed into the corresponding recipient structures. The anastomoses were secured with 6.0 polypropylene ties. The implantation time was standardized at 15 minutes. The trachea of the transplanted lung was connected to a second ventilator (Model RV5; Voltek Enterprises Inc, Toronto, Ontario, Canada). This ventilator delivered constant inspiratory flow by allowing inspiratory gas to the lungs from a high-pressure source (20-50 pounds per square inch) through a high-resistance capillary tube. The recipient's native right lung and left transplanted lung were thus ventilated independently. A volume recruitment maneuver of $35 \mathrm{~cm} \mathrm{H}_{2} \mathrm{O}$ for 5 seconds was delivered to the transplanted lung before initiation of reperfusion. Reperfusion was then started by unclamping the PV, followed by the PA. The transplanted lung was reperfused for 3 hours.

\section{Experimental Protocol}

Animals were randomized into 2 groups defined by the ventilatory strategy of the transplanted lung after reperfusion: (1) in the conventional mechanical ventilation group (conventional group, $\mathrm{n}=5$ ) the transplanted lung was ventilated with a $\mathrm{V}_{\mathrm{T}}$ equal to $50 \%$ of the inspiratory capacity of the left lung and low PEEP, and (2) in the minimal mechanical stress ventilation group (minimal stress group, $\mathrm{n}=5$ ) the transplanted lung was ventilated with a $\mathrm{V}_{\mathrm{T}}$ equal to $20 \%$ of the inspiratory capacity of the left lung, and PEEP was adjusted to maintain values of stress index between 0.9 and 1.1 (see details below). Stress index values were checked after a volume recruitment maneuver of $35 \mathrm{~cm} \mathrm{H}_{2} \mathrm{O}$ for 5 seconds every 30 minutes during the 3 -hour ventilatory time, and PEEP was adjusted accordingly. In both groups the right native lung was independently ventilated with a $\mathrm{V}_{\mathrm{T}}$ of $5 \mathrm{~mL} / \mathrm{kg}$ and a PEEP of $3 \mathrm{~cm}$
$\mathrm{H}_{2} \mathrm{O}$ during the 3-hour reperfusion period. The inspiratory capacity of the left lung had been determined in a pilot study by manually inflating the left lung up to a plateau pressure (Pplat) of $30 \mathrm{~cm} \mathrm{H}_{2} \mathrm{O}$ after a volume recruitment maneuver of $35 \mathrm{~cm} \mathrm{H}_{2} \mathrm{O}$ for 5 seconds.

The presence of air leaks from the transplanted lung was checked every 30 minutes during measurement of the end-inspiratory Pplat. When a leak was found $(n=2)$, the animals were excluded from the study and replaced in the randomization to obtain 5 animals per group. Blood pressure was continuously monitored, and arterial blood gas analysis from both lungs was performed every hour during the 3-hour reperfusion period. At the end of the reperfusion period, arterial blood gases from the transplanted lung were measured after occluding the right PA with a microsurgical aneurysm clamp under direct vision. The animals were then killed with an overdose of sodium pentobarbital, and the transplanted lung was immediately removed and divided into 3 parts. The inferior and superior third were snap-frozen in liquid nitrogen and stored at $-70^{\circ} \mathrm{C}$ for determination of cytokines. The middle third was fixed in $10 \%$ buffered formalin for at least 24 hours and then submitted for pathologic examination.

\section{Measurements}

Inspiratory flow was determined as the pressure drop across the capillary tube of the ventilator. This pressure signal was calibrated with the same gas mixture used to ventilate the animal, and the linearity of the pressure transducer was confirmed to be within the range of flow used in the study. $\mathrm{P}_{\mathrm{L}}$ was equal to the airway opening pressure (PAO) in the transplanted lung because of the open thoracotomy. PAO was measured proximal to the endotracheal tube of the transplanted lung with a pressure transducer. Inspiratory flow and PAO were displayed and collected (ICU-Lab; KleisTEK Advanced Electronic Systems, Bari, Italy) on a laptop computer equipped with a 12-bit analog-digital acquisition board (DAQ card 700; National Instrument, Austin, Tex) at a sampling rate of $600 \mathrm{~Hz}$. PEEP was measured at the end of a 3- to 4-second end-expiratory occlusion (PEEP $=$ external PEEP + auto PEEP). Pplat was measured at the end of a 3- to 4-second end-inspiratory occlusion. $\mathrm{V}_{\mathrm{T}}$ was calculated by integration of the inspiratory flow. A volume recruitment maneuver of $35 \mathrm{~cm} \mathrm{H}_{2} \mathrm{O}$ for 5 seconds was performed before all measurements to normalize volume history.

\section{Measurement of Stress Index}

During constant flow inflation, the P-t relation on a breath-bybreath basis can be described by a power equation ${ }^{15,16}$ :

$$
\mathrm{P}_{\mathrm{L}}=\mathrm{a} \cdot \mathrm{t}^{\mathrm{b}}+\mathrm{c}
$$

where $\mathrm{P}_{\mathrm{L}}$ is inspiratory PAO, $\mathrm{t}$ is inspiratory time, and the coefficients $\mathrm{a}, \mathrm{b}$, and $\mathrm{c}$ are constants. The coefficient $\mathrm{b}$ is a dimensionless number that describes the shape of the P-t curve and can be considered to be an index of lung stress, which we have termed the stress index. For stress index values of less than 1, the P-t curve presents a downward concavity, corresponding to a static P-V curve with a distinct LIP and a continuous increase in compliance, suggesting mechanical stress caused by tidal recruitment-derecruitment of alveolar units. For stress index values of greater than 1, the P-t curve presents an upward concavity corresponding to a static P-V curve with a distinct UIP and a continuous reduction in compliance, suggesting mechanical stress caused by overdistention 
of the alveolar units. For stress index values equal to 1 , the P-t curve is straight, indicating neither overdistention nor derecruitment and suggesting minimal mechanical stress. ${ }^{17}$ The beginning and end of each inspiration was determined from the zero crossing points of the flow curve. Inspiratory flow and $P_{L}$ signals were averaged on a breath-by-breath basis and over 2- to 3-minute periods every 5 minutes. The power equation was then fit to the resulting mean $\mathrm{P}_{\mathrm{L}}$. The curve-fitting procedure was applied to the $\mathrm{P}_{\mathrm{L}}$ data points corresponding to the constant part of the mean inspiratory flow. The curve-fitting procedure included only data points obtained from $50 \mathrm{~ms}$ after the beginning of the square wave in inspiratory flow until $50 \mathrm{~ms}$ before the end of flow to ensure that the on-flow and off-flow transients did not skew the results. These values were chosen on the basis of a series of preliminary experiments performed to identify the opening and closing time of the solenoid valve used on the rat ventilator and to verify that inspiratory flow remained constant in the pressure range used in the current study. Stress index values were displayed on the computer screen.

\section{Study End Points}

Respiratory mechanics. Elastance was calculated every 30 minutes during the 3-hour reperfusion period as follows:

$$
(\text { Pplat }- \text { PEEPt }) / \mathrm{V}_{\mathrm{T}}
$$

Gas exchange. Blood samples were taken from the right carotid artery every hour during the 3-hour reperfusion period. In addition, one sample was taken after the right PA was occluded for 10 minutes at the end of the reperfusion period to analyze gas exchange from the transplanted lung only.

Lung tissue cytokines. Frozen tissues from the inferior and superior third of the lung were homogenized and incubated at $4{ }^{\circ} \mathrm{C}$ in cell lysis buffer containing $10 \mathrm{mmol} / \mathrm{L} \mathrm{N}$-2-hydroxyethylpiperazine-N-2-ethanesulfonic acid ( $\mathrm{pH} 7.9), 10 \mathrm{mmol} / \mathrm{L} \mathrm{KCL}, 0.1$ $\mathrm{mmol} / \mathrm{L}$ ethylenediamine tetraacetic acid, $0.1 \mathrm{mmol} / \mathrm{L}$ ethyleneglycol-bis-( $\beta$-aminoethylether)-n, $\mathrm{n}^{\prime}$-tetraacetic acid, $1 \mathrm{mmol} / \mathrm{L} \mathrm{di-}$ thiothreitol, $0.5 \mathrm{mmol} / \mathrm{L}$ phenylmethylsulfonyl fluoride, and $0.6 \%$ octylphenoxy-polyethoxy-ethanol (Nonidet P-40). ${ }^{18}$

Homogenates were then sonicated and centrifuged at 12,000 rpm for 10 minutes at $4{ }^{\circ} \mathrm{C}$. Supernatants were assayed in duplicate by using specific ELISA kits for rat tumor necrosis factor $\alpha$ (TNF- $\alpha$ ), rat interleukin 6 (IL-6), and rat macrophage inflammatory protein 2 (MIP-2), according to the manufacturer's instructions. Specific Cytoscreen Immunoassay Kits (BioSource International, Inc, Camarillo, Calif) were used for all cytokines. The optical density of each well was read at $450 \mathrm{~nm}$ with an NM-600 microplate reader (Dynatech Laboratories, Chantilly, Va). The final concentration was calculated by converting the optical density readings against a standard curve. The protein content was determined by using the Bradford method. ${ }^{19}$

Pathologic lung injury score. The lung tissue fixed in $10 \%$ formalin was processed for histologic analysis and embedded in paraffin. Thereafter, each section was sliced to $5 \mu \mathrm{m}$ and was stained with hematoxylin and eosin. The specimens were then submitted for histologic analysis by a pathologist (J.B.M.), who was blinded as to the type of ventilation protocol used. Bronchiolar epithelial lesions (necrosis and epithelial sloughing) were quantified by using a modification of the method of Nilsson and col- leagues, ${ }^{20}$ as described previously. ${ }^{21}$ In each section the total respective number of membranous and respiratory bronchioles were counted. An injury score for each airway type was obtained as the percentage of injured airways of each airway type. The parenchyma was graded by using the modified quantitive histologic technique of Silberschmid and coworkers. ${ }^{22}$ The presence and extent of interstitial cellular infiltrate, alveolar hyaline membrane formation, alveolar edema, and cellular exudates were assessed. From each section, 10 random areas were examined at medium magnification $(250 \times)$. By using a square lattice test grid $(2 \times 2)$, a separate score from 0 to 100 was determined for each histologic parameter by counting the number of squares showing the change indicated and expressing this number as a percentage of the maximal positive score.

\section{Statistical Analysis}

All data are expressed as mean values \pm SD. A 1-way analysis of variance was used to determine statistical significance. For differences in elastance in the transplanted lung over the 3-hour reperfusion period, a 2-way analysis of variance was used. The Graphpad software package (Graphpad Software, Inc, San Diego, Calif) was used for all statistical analyses.

\section{Results}

All animals included in the study survived and remained hemodynamically stable during the reperfusion period; no significant differences were observed in the systemic blood pressure between groups at any time points. One animal in each group was excluded because of the development of air leaks during the reperfusion period. Both animals were replaced in the randomization, and 5 animals per group were included in the final data analysis. Donor and recipient rats were size matched.

\section{Ventilation and Respiratory Mechanics}

The ventilation protocols of the transplanted lungs resulted in marked differences in PEEP and Pplat values between the groups (Figure 1). Elastance remained significantly lower in the minimal stress ventilation group than in the conventional ventilation group, indicating less lung injury in the minimal stress ventilation group (Figure 2).

\section{Gas Exchange}

Systemic blood gases representing gas exchange from both lungs were not significantly different between groups after 0,1 , and 2 hours of reperfusion. At 3 hours, $\mathrm{PaO}_{2}$ was significantly higher in the minimal stress ventilation group than in the conventional ventilation group $(328 \pm 18.3$ vs $273 \pm 21.9 \mathrm{~mm} \mathrm{Hg}$, respectively; $P=.05)$, but the $\mathrm{PaCO}_{2}$ was not significantly different between the 2 groups (55 \pm 1.8 vs $50 \pm 1.4 \mathrm{~mm} \mathrm{Hg}$, respectively; $P=.08$ ). The $\mathrm{PaO}_{2}$ from the transplanted lung (measured after clamping the right PA) was also significantly higher in the minimal stress ventilation group than in the conventional ventilation group at the end of the reperfusion period (392 \pm 46 vs $224 \pm 52$ $\mathrm{mm} \mathrm{Hg}$, respectively; $P=.0006$; Figure 3 ). 

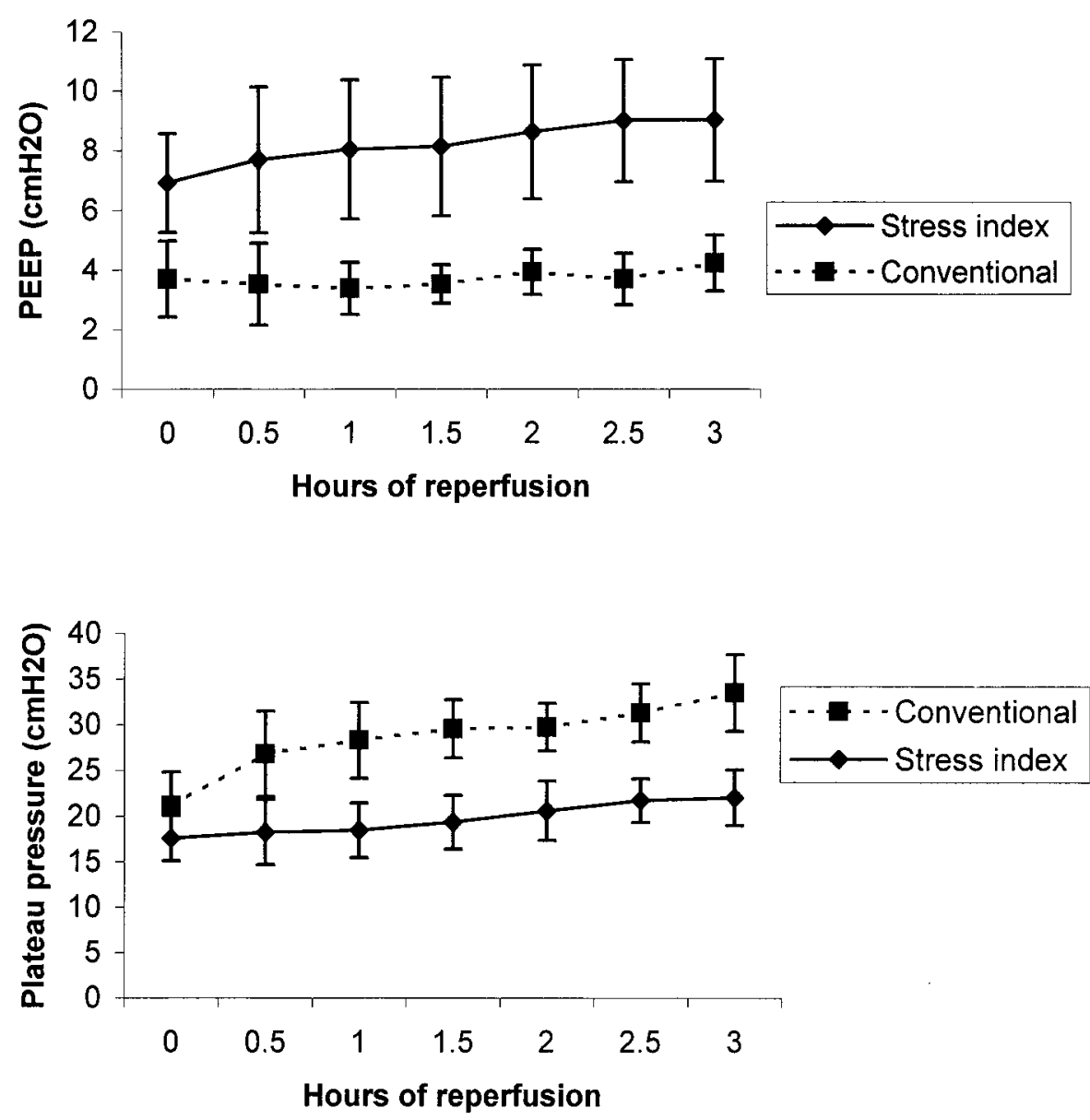

Figure 1. There were marked differences in PEEP and Pplat between the conventional mechanical ventilation group (conventional) and the minimal mechanical stress ventilation group (stress index).

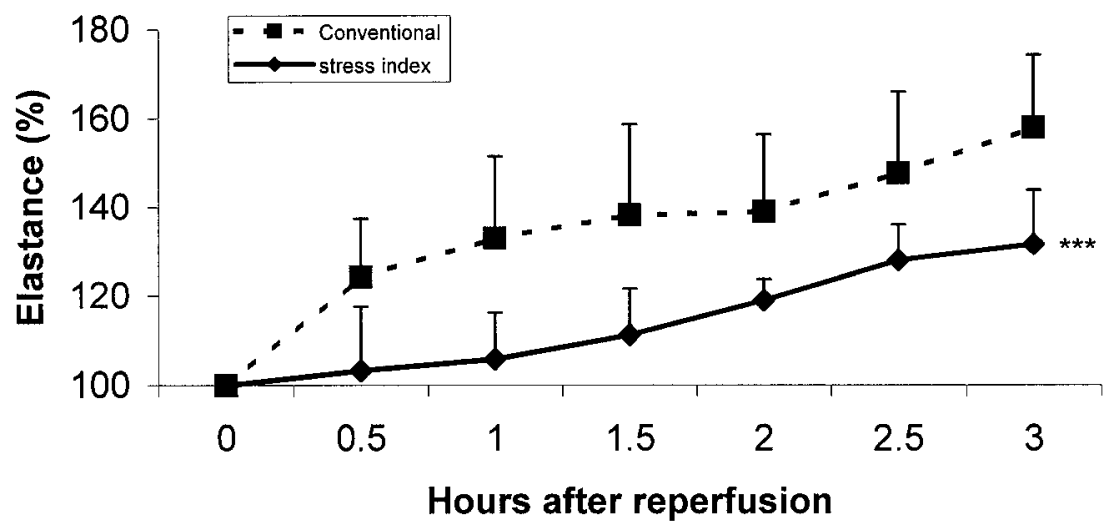

Figure 2. Elastance, which directly correlates with the degree of lung injury, was significantly higher in the conventional mechanical ventilation group (conventional) than in the minimal mechanical stress ventilation group (stress index, ${ }^{* * *} \boldsymbol{P}<.0001$ vs the conventional mechanical ventilation group).

Lung Tissue Cytokines

MIP-2 and IL-6 levels in lung tissue were significantly higher in the conventional ventilation group than in the minimal stress ventilation group. The levels of TNF- $\alpha$ were not significantly different between groups, although there was a trend toward higher levels in the conventional ventilation group (Figure 4). 

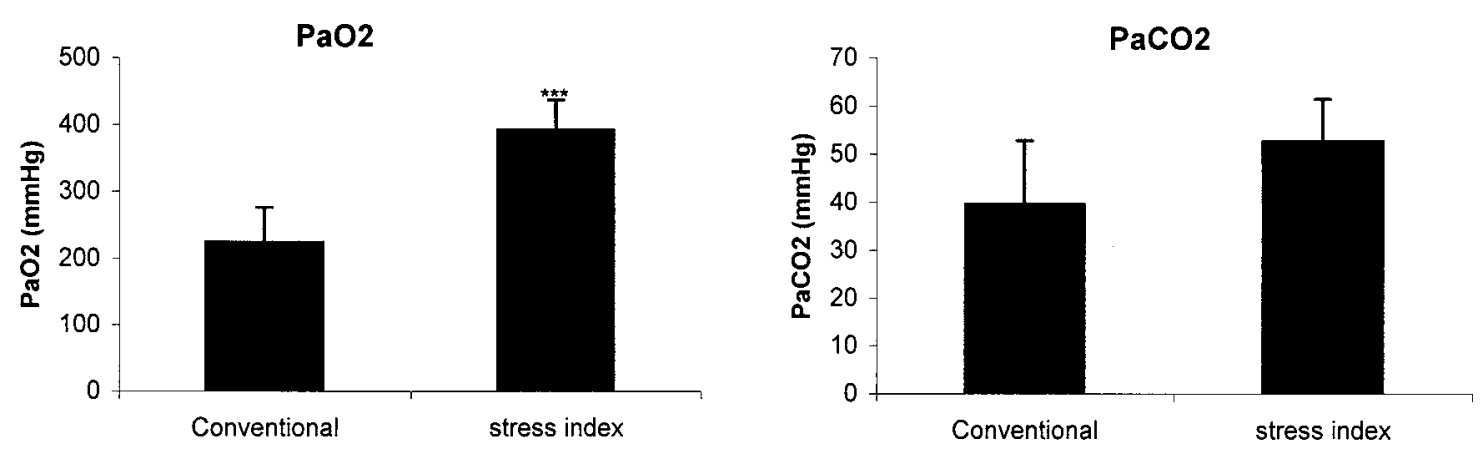

Figure 3. $\mathrm{PaO}_{2}$ of the transplanted lung was significantly higher in the minimal mechanical stress ventilation group (stress index) than in the conventional mechanical ventilation group (conventional; ${ }^{* *} \boldsymbol{P}<.0006$ vs the conventional mechanical ventilation group). The difference between the $\mathrm{PaCO}_{2}$ was not significantly different between groups $(P=.1)$.

IL-6

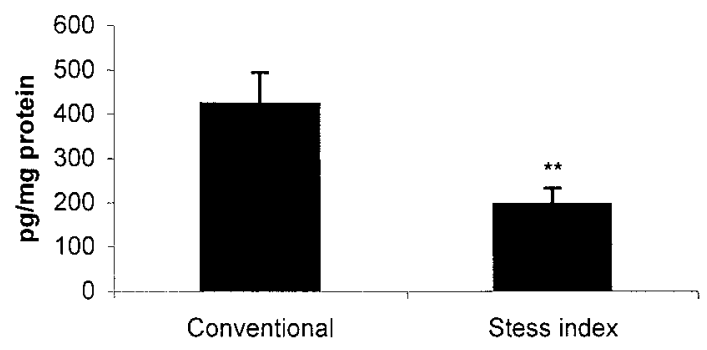

MIP-2

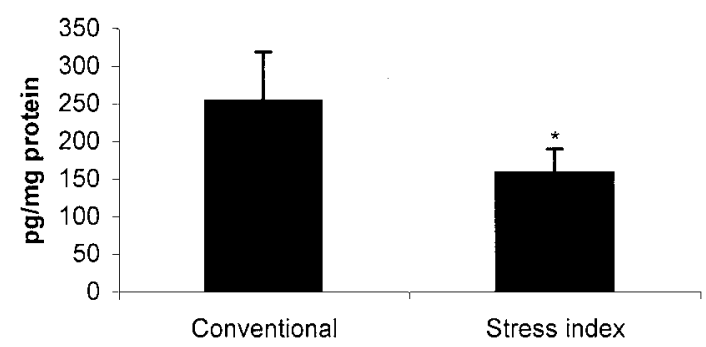

TNF-alpha

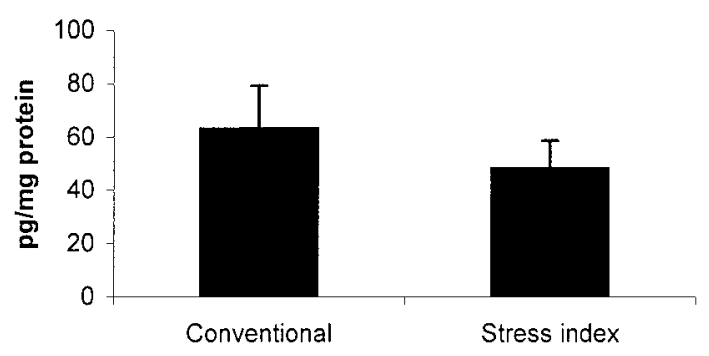

Figure 4. The proinflammatory cytokines IL-6 and MIP-2 were significantly lower in the minimal mechanical stress ventilation group (stress index) than in the conventional mechanical ventilation group (conventional; ${ }^{*} \boldsymbol{P}<.05$ and ${ }^{* *} \boldsymbol{P}<.01$ vs the conventional mechanical ventilation group). TNF- $\alpha$ was not significantly different between groups $(P=.1)$.

\section{Pathologic Lung Injury Score}

Pathologic findings from the transplanted lung in the conventional ventilation group showed a significantly higher rate of hyaline membrane formation than in the minimal stress ventilation group $(25 \% \pm 18 \%$ vs $6 \% \pm 4 \%$, respectively; $P=.04$; Figure 5). In addition, membranous and respiratory bronchioles were injured only in the conventional ventilation group $(12 \% \pm 14 \%$ and $21 \% \pm 12 \%$, respectively, vs $0 \%$ in the minimal stress ventilation group).
The interstitial cellular infiltrate, alveolar edema, and cellular exudates were not significantly different between groups. However, there was a trend toward lower cellular exudates in the minimal stress ventilation group $(3 \% \pm 2 \%$ vs $9 \% \pm 9 \%$ in the conventional ventilation group, $P=.1$ ).

\section{Discussion}

This study demonstrates that in an in vivo rat single lung transplant model the ventilatory strategy used in the early 
phase of reperfusion can have a major effect on the deterioration of lung mechanics, gas exchange, production of pulmonary cytokines, and lung pathology. In addition, we have shown that a protective ventilatory strategy on the basis of analysis of the P-t curve during constant flow ventilation and the online assessment of stress index can attenuate I-R injury after lung transplantation.

In this left single lung transplant model we used independent ventilation for the right and left lungs to better control the $\mathrm{V}_{\mathrm{T}}$ and PEEP applied to each lung and to evaluate the effect of different ventilatory strategies on the transplanted lung. The right native lung was ventilated with the same ventilation protocol in both groups, and the left (transplanted) lung was ventilated with different ventilatory strategies: a conventional mechanical ventilation group consisting of $\mathrm{V}_{\mathrm{T}}$ equal to $50 \%$ of the inspiratory capacity of the left lung and low PEEP and a minimal mechanical stress ventilation group consisting of $\mathrm{V}_{\mathrm{T}}$ equal to $20 \%$ of the inspiratory capacity of the left lung and PEEP adjusted to obtain a stress index of $1 \pm 0.1$.

We found that the transplanted lung ventilated with conventional mechanical ventilation had a significant increase in elastance with higher cytokine concentrations and greater pathologic changes compared with the transplanted lung in the minimal stress ventilation group. The striking morphologic differences between the 2 groups (Figure 5) demonstrate the potential effect of mechanical ventilation on the development of lung injury after reperfusion. These findings are consistent with reports analyzing the effects of mechanical ventilation on lungs injured with other insults than I-R and are likely related to alteration in the alveolar-endothelial barrier, defects in surfactant production, or both. ${ }^{8,21,23,24}$

The proinflammatory cytokines IL-6 and MIP-2 (rodent homologue of human IL-8) were significantly higher in the lungs ventilated with the injurious ventilatory strategy. Levels of IL- 6 and IL- 8 have been shown to correlate with lung function and the development of I-R injury in patients after lung transplantation. ${ }^{25-27}$ We observed, however, that TNF- $\alpha$ levels were not significantly different between the 2 groups. This finding might reflect different timing in the release of cytokines after lung transplantation. Indeed, in contrast to IL-6 and IL-8, which are released for a period of several hours after lung transplantation, TNF- $\alpha$ has been shown to peak during the ischemic period or immediately after reperfusion in lung transplantation. ${ }^{25,28}$ Hence its release might have been influenced to a lower degree by the ventilatory strategy in our model.

The static $\mathrm{P}-\mathrm{V}$ relation of the respiratory system has been extensively studied in animal models and in patients with ALI. ${ }^{10,21,29}$ The P-V curve is characterized by an LIP and a UIP. The LIP represents the average critical opening pressure above which alveolar units start to reopen. It has been suggested that the end-expiratory volume pressure should
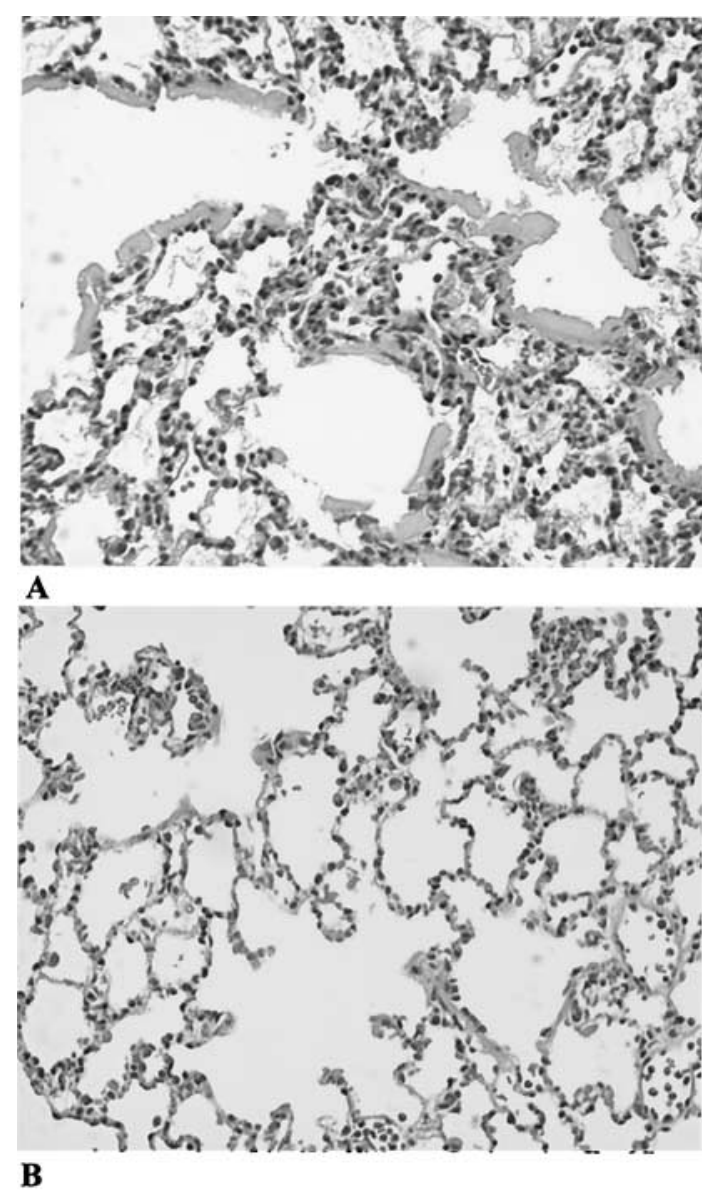

Figure 5. Hematoxylin-and-eosin staining showing the formation of hyaline membranes in the conventional mechanical ventilation group and its absence in the minimal mechanical stress ventilation group.

be maintained above the LIP to avoid cyclic end-expiratory alveolar collapse. The UIP indicates the P-V values above which overdistention starts to occur, and $V_{T}$ should be limited to maintain end-inspiratory volume and pressure at a level lower than the UIP. Recent clinical data suggest that the static P-V curve might be useful in setting values of PEEP and $V_{T}$ to minimize VILI and improve outcomes. ${ }^{10,11}$ Nevertheless, application of the static P-V curve is limited, and there are a number of issues that challenge the utility of this approach, including the following: (1) the lung is often not fully recruited, even if PEEP is above the LIP, and (2) measurement of $\mathrm{P}-\mathrm{V}$ curves cannot be easily accomplished in clinical practice. The results of the present study suggest that stress index strategy might represent an alternative to the static $\mathrm{P}-\mathrm{V}$ curve to determine lung protection ventilatory parameters. Modern ventilators are able to deliver excellent square-wave inspiratory flow profiles and are also equipped with monitoring tools that enable online, dynamic P-t curves. This implies that PEEP and $\mathrm{V}_{\mathrm{T}}$ could be set contin- 
uously on virtually a breath-by-breath basis to minimize VILI by maintaining values of stress index close to 1 .

In conclusion, this study demonstrates for the first time that mechanical ventilation can have a major effect on the development of lung injury in an animal model of lung transplantation. This observation implies that VILI might be an underrecognized phenomenon that contributes significantly to I-R injury after lung transplantation. Therefore protective ventilatory strategies could potentially lead to improved outcomes after lung transplantation. The stress index strategy could be a novel option for protective ventilatory management of the transplanted lung. Further studies are required to determine the optimal protective ventilatory strategy in the setting of lung transplantation.

\section{References}

1. Hosenpud JD, Benett LE, Keck BM, et al. The registry of the International Society for Heart and Lung Transplantation: seventeenth official report-2000. J Heart Lung Transplant. 2000;19:909-31.

2. Christie JD, Bavaria JE, Palevsky HI, et al. Primary graft failure following lung transplantation. Chest. 1998;114:51-60.

3. King RC, Binns OAR, Rodriguez F, et al. Reperfusion injury significantly impacts clinical outcome after pulmonary transplantation. Ann Thorac Surg. 2000;69:1681-5.

4. Qayumi AK, Nikbakht-Sangari M, Godin DV, et al. The relationship of ischemia-reperfusion injury of transplanted lung and up-regulation of major histocompatibility complex II on host peripheral lymphocytes. J Thorac Cardiovasc Surg. 1998;115:978-89.

5. Crocetti JG, O'Brien OB, Furukawa S, et al. The impact of reperfusion injury on the long term development of obliterative bronchiolitis (OB) in lung transplantation [abstract]. Am J Respir Crit Care Med. 2000; 161:A507.

6. Dos Santos CC, Slutsky AS. Mechanisms of ventilator-induced lung injury: a perspective. J Appl Physiol. 2000;89:1645-55.

7. Slutsky AS. Lung injury caused by mechanical ventilation. Chest. 1999;116:9S-15S.

8. Tremblay L, Valenza F, Ribeiro SP, Li J, Slutsky AS. Injurious ventilatory strategies increases cytokines and c-fos m-RNA expression in an isolated rat lung model. J Clin Invest. 1997;99:944-52.

9. Pelosi P, Goldner M, McKibben A, et al. Recruitment and derecruitment during acute respiratory failure. An experimental study. Am J Respir Crit Care Med. 2001;164:122-30.

10. Amato MB, Barbas CSV, Medeiros DM, et al. Effect of a protectiveventilation strategy on mortality in the acute respiratory distress syndrome. N Engl J Med. 1998;338:347-54.

11. Ranieri VM, Suter PM, Tortorella C, et al. Effect of mechanical ventilation on inflammatory mediators in patients with acute respiratory distress syndrome. JAMA. 1999;282:54-61.

12. The Acute Respiratory Distress Syndrome Network. Ventilation with lower tidal volumes as compared with traditional tidal volumes for acute lung injury and the acute respiratory distress syndrome. $N$ Engl J Med. 2000;342:1301-8.

13. Harris RS, Hess DR, Venegas JG. An objective analysis of the pressure-volume curve in the acute respiratory distress syndrome. Am J Respir Crit Care Med. 2000;161:432-9.

14. Rimensberger PC, Cox PN, Frndova H, Bryan AC. The open lung during small tidal volume ventilation: concepts of recruitment and "optimal" positive end-expiratory pressure. Crit Care Med. 1999;27: 1946-52.

15. Ranieri VM, Giuliani R, Fiore T, Dambrosio M, Milic-Emili J. Volume-pressure curve of the respiratory system predicts effects of PEEP in ARDS: "occlusion" versus "constant flow" technique. Am J Respir Crit Care Med. 1994;149:19-27.

16. Bates JHT, Rossi A, Milic-Emili J. Analysis of the behavior of the respiratory system with constant inspiratory flow. J Appl Physiol. 1985;58:1840-8.

17. Ranieri VM, Zhang H, Mascia L, et al. Pressure-time curve predicts minimally injurious ventilatory strategy in an isolated rat lung model. Anesthesiology. 2000;93:1320-8.

18. Gilbert SP, Johnson KA. Pre-steady-state kinetics of the microtubulekinesin ATPase. Biochemistry. 1994;33:1951-60.

19. Bradford MM. A rapid and sensitive method for the quantitation of microgram quantities of protein utilizing the principle of protein-dye binding. Anal Biochem. 1976;72:248-54.

20. Nilsson R, Grossmann G, Robertson B. Lung surfactant and the pathogenesis of neonatal bronchiolar lesions induced by artificial ventilation. Pediatr Res. 1978;12:249-55.

21. Muscedere JG, Mullen JB, Gan K, Slutsky AS. Tidal ventilation at low airway pressures can augment lung injury. Am J Respir Crit Care Med. 1994;149:1327-34.

22. Silberschmid M, Szczepanski KP, Lund C. Hemorrhagic shock in dogs without major pulmonary changes. Eur Surg Res. 1975;7:10-22.

23. Chiumello D, Pristine G, Slutsky AS. Mechanical ventilation affects local and systemic cytokines in an animal model of acute respiratory distress syndrome. Am J Respir Crit Care Med. 1999;160:109-16.

24. Ito Y, Veldhuizen RA, Yao LJ, McCaig LA, Bartlett AJ, Lewis JF. Ventilation strategies affect surfactant aggregate conversion in acute lung injury. Am J Respir Crit Care Med. 1997;155:493-9.

25. de Perrot M, Sekine Y, Fischer S, et al. Interleukin-8 release during early reperfusion predicts graft function in human lung transplantation. Am J Respir Crit Care Med. 2002;165:211-5.

26. Fisher AJ, Donnelly SC, Hirani N, et al. Elevated levels of interleukin-8 in donor lungs is associated with early graft failure after lung transplantation. Am J Respir Crit Care Med. 2001;163:259-65.

27. Pham SM, Yoshida Y, Aeba R, et al. Interleukin-6, a marker of preservation injury in clinical lung transplantation. J Heart Lung Transplant. 1992;11:1017-24.

28. Serrick C, Adoumie R, Giaid A, Shennib H. The early release of interleukin-2, tumor necrosis factor- $\alpha$ and interferon- $\gamma$ after ischemia reperfusion injury in the lung allograft. Transplantation. 1994;58: 1158-62.

29. Ranieri VM, Brienza N, Santostasi S, et al. Impairment of lung and chest wall mechanics in patients with acute respiratory distress syndrome: the role of abdominal distention. Am J Respir Crit Care Med. 1997;156:1082-91. 